\title{
4 Entwicklung einer Kostensensiblen Leitlinie zur Implantation eines ICDs bei Patienten mit chronischer Herzinsuffizienz
}

\author{
Daniela Gartner-Freyer, Janine Biermann, Kirstin Börchers, \\ Petra Schnell-Inderst, Jürgen Wasem und Anja Neumann
}

\subsection{Hintergrund}

Bei begrenzten finanziellen Ressourcen im deutschen Gesundheitswesen führt eine Ausgabenexpansion durch den biomedizinischen Fortschritt zu erheblichen Verteilungsproblemen. Da die Einschränkung von Gesundheitsleistungen vielen Akteuren mittelfristig unumgänglich erscheint, findet in Deutschland eine zunehmende Diskussion insbesondere zum Thema Rationierung im Gesundheitswesen statt. Dabei lassen sich zwei Formen unterscheiden: Explizite Rationierung basiert auf Entscheidungen oberhalb der individuellen Arzt-Patienten-Beziehung, während bei einer impliziten Rationierung die Allokationsentscheidungen auf der Mikroebene stattfinden. Es erscheint sinnvoll, Rationierungen primär auf einer expliziten Ebene durchzuführen, um das direkte Arzt-Patienten-Verhältnis zu entlasten. (ZEKO o.V. 2007)

Methodisch stellt die Erarbeitung von KSLL eine Möglichkeit zur Anwendung expliziter Rationierung dar: Evidenzbasierte medizinische Leitlinien werden durch subgruppenspezifische Kosteneffektivitätsdaten ergänzt, mittels derer Empfehlungen zur expliziten Rationierung gegeben werden können (Eccles u. Mason 2001).

Im Rahmen dieser Arbeit wird beispielhaft die Entwicklung einer KSLL zur Implantation eines implantierbaren Cardioverters/Defibrillators (ICD) in der 
4 Entwicklung einer Kostensensiblen Leitlinie zur Implantation eines ICDs bei Patienten mit chronischer Herzinsuffizienz

Primärprävention des plötzlichen Herztodes bei Patienten mit chronischer Herzinsuffizienz untersucht.

\subsection{Methodik}

Zunächst wurden national und international verfügbare, thematisch einschlägige Leitlinien und Versorgungsstandards zur chronischen Herzinsuffizienz/ Implantation von ICDs identifiziert und daraufhin untersucht, inwieweit Kosten-Effektivitäts-Erwägungen bereits explizit oder implizit eingeflossen sind. Daran schloss sich die Analyse der empirischen Evidenz zur Effektivität und Kosten-Effektivität für den Einsatz von ICDs in der Primärprävention kardialer Rhythmusstörungen bei chronischer Herzinsuffizienz an. Des Weiteren wurde eine Identifikation von geeigneten Subgruppen in Bezug auf die Kosteneffektivität zur Aufarbeitung in KSLL vorgenommen.

Es wurde, wie im Folgenden ausgeführt, exemplarisch eine KSLL mit Beschreibung des Krankheitsbildes und Hintergrundes sowie einer Zusammenfassung zur Effektivität und Kosteneffektivität dargestellt.

\subsubsection{Literaturrecherche}

\section{Klinische Leitlinien}

Das Ziel der Leitlinienrecherche bestand in der Identifizierung einer aktuellen, evidenzbasierten und auf den deutschen Kontext übertragbaren Leitlinie zur Implantation eines ICDs im Rahmen der Primärprävention zur Verhinderung des plötzlichen Herztodes bei Patienten mit chronischer Herzinsuffizienz.

Mittels manueller Recherchen in Leitliniendatenbanken sowie bei Fachgesellschaften und in den bibliographischen Datenbanken MEDLINE und EMBASE wurde nach thematisch einschlägigen Leitlinien zum Thema ICD im Rahmen der Primärprävention gesucht.

Die Suche nach relevanten Leitlinien wurde in den in Tabelle 8 dargestellten Quellen durchgeführt.

Als Einschlusskriterien der Literaturrecherche wurden klinische Leitlinien zu 1. Patienten $\geq 18$ Jahre, 2. zur Primärprävention des plötzlichen Herztodes bei Patienten mit chronischer Herzinsuffizienz, 3. mittels eines ICDs definiert. Als 4. Einschlusskriterium wurde eine sprachliche Eingrenzung auf Publikationen in deutscher oder englischer Sprache festgelegt. Als Ausschlusskriterium wurde das Erscheinungsjahr definiert, wobei alle Leitlinien, die vor 2006 erschienen sind, aus Gründen der Aktualität ausgeschlossen wurden. Ein weiteres Kriterium zum Ausschluss stellten Mehrfachpublikationen ohne relevante Zusatzinformationen dar. 


\section{Tab. 8 Datenbanken zur Leitlinienrecherche}

\begin{tabular}{|c|c|}
\hline Quelle & Kommentar \\
\hline $\begin{array}{l}\text { Leitliniendatenbanken, } \\
\text { Fachgesellschaften }\end{array}$ & $\begin{array}{l}\text { http://www.sign.ac.uk/ } \\
\text { http://www.escardio.org/ } \\
\text { http://www.hebw.cf.ac.uk/index.html } \\
\text { http://www.leitlinien.de }{ }^{1} \\
\text { http://www.versorgungsleitlinien.de/themen }{ }^{1} \\
\text { http://www.bundesaerztekammer.de } \\
\text { http://www.awmf.org } \\
\text { http://www.evidence.de/ } \\
\text { http://www.leitlinien.net }{ }^{2} \\
\text { http://www.g-i-n.net } \\
\text { http://www.gacguidelines.ca } \\
\text { http://www.nzgg.org.nz } \\
\text { http://www.guideline.gov/ } \\
\text { http://www.heart.org/ } \\
\text { http://www.nice.org.uk/ } \\
\text { http://www.ahrq.gov/3 } \\
\text { http://leitlinien.dgk.org/ } \\
\text { http://www.cma.ca/ }\end{array}$ \\
\hline bibliographische Datenbanken & $\begin{array}{l}\text { Medical Literature Analysis and Retrieval System Online } \\
\text { (MEDLINE) } \\
\text { Excerpta Medica Database (EMBASE) }\end{array}$ \\
\hline
\end{tabular}

Die gefundenen Literaturstellen wurden in einem zweistufigen Screening überprüft: In der ersten Stufe wurden mittels der definierten Einschlusskriterien anhand von Titel und Abstract die identifizierten Literaturstellen von zwei voneinander unabhängigen Reviewern vorselektiert (1. Screening). Auf der zweiten Stufe wurden die Volltexte ebenfalls von zwei voneinander unabhängigen Reviewern auf die inhaltliche Relevanz überprüft (2. Screening).

Für die Bewertung der klinischen Leitlinien erfolgte - wiederum von zwei voneinander unabhängigen Reviewern - eine Untersuchung mithilfe des Deutschen Instruments zur methodischen Leitlinien-Bewertung (DELBI)7, welches

7 Checkliste basiert auf den Vorgaben der German Scientific Working Group Technology Assessment for Health Care (2000) 
4 Entwicklung einer Kostensensiblen Leitlinie zur Implantation eines ICDs bei Patienten mit chronischer Herzinsuffizienz

die Bewertung der methodischen Qualität medizinischer Leitlinien ermöglichen soll.

Es wurde die aktuellste und mit dem höchsten Summenwert nach DELBI (Beyer et al. 2008) bewertete Leitlinie als Grundlage der Entwicklung einer KSLL ausgewählt.

\section{Medizinische Effektivität und Kosteneffektivität}

Mittels einer systematischen Literaturrecherche wurde die empirische Evidenz zur Effektivität und Kosteneffektivität des Einsatzes von ICDs in der Primärprävention des plötzlichen Herztodes bei Patienten mit Herzinsuffizienz analysiert. In diesem Zusammenhang wurde untersucht, inwiefern Daten zur Kosteneffektivität in spezifischen Patientensubgruppen vorhanden sind.

In einem ersten Schritt wurde in 2007 zunächst eine systematische Literaturrecherche zur Effektivität und Kosteneffektivität des Einsatzes von ICD im Rahmen der Primärprävention beschränkt auf HTAs durchgeführt, die 2009 durch eine Nachrecherche ergänzt wurde.

Die durchsuchten Datenbanken mit den entsprechend verwendeten Suchbegriffen können der Tabelle 9 entnommen werden.

Als Einschlusskriterien der Literaturrecherche wurden Publikationen zu 1. Patienten $\geq 18$ Jahre, 2. zur Primärprävention des plötzlichen Herztodes bei Patienten mit chronischer Herzinsuffizienz, 3. mittels eines ICDs definiert. Als 4. Einschlusskriterium wurde eine sprachliche Eingrenzung auf Publikationen in deutscher oder englischer Sprache festgelegt. 5. Eingeschlossen wurden ausschließlich HTAs. Spezifische Ausschlusskriterien bestanden nicht.

Die definierten Einschlusskriterien wurden benutzt, um anhand von Titel und Abstract die identifizierten Literaturstellen von zwei Mitarbeitern unabhängig voneinander vorzuselektieren (1. Screening). In einem zweiten Schritt wurden

\section{Tab. 9 Auflistung der durchsuchten Datenbanken mit entsprechenden Suchbegriffen}

$\begin{array}{ll}\text { Datenbanken } & \text { verwendete Suchbegriffe } \\ \text { http://nhscrd.york.ac.uk/ } & \text { ICD } \\ \text { http://www.cochrane.de } & \text { Defibrillator } \\ \text { www.dimdi.de } & \text { Implantable defibrillator } \\ \text { www.egms.de } & \text { Icd } \\ \text { www.msac.gov.au } & \text { Implantable defibrillator } \\ \text { www.cadth.ca } & \text { Defibrillators, implantable } \\ \text { www.iqwig.de } & \\ \text { www.kbv.de } & \\ \text { www.nice.org.uk } & \end{array}$


die HTAs anhand der Volltextversionen gescreent (2. Screening). Ausschlussgründe wurden festgehalten. Die Selektionsschritte und die Anzahl der einund ausgeschlossenen HTAs auf den verschiedenen Selektionsebenen wurden in einem Flussdiagramm dargestellt.

Die in die Evaluation eingeschlossenen Publikationen wurden anhand der Checkliste 1a [HTAs], ıb [systematische Reviews, Metaanalysen] geprüft. Die aktuellsten und qualitativ hochwertigsten HTAs wurden als Grundlage der weiteren Analysen zur medizinischen Effektivität und Kosteneffektivität verwendet.

Aufbauend auf der Suchstrategie des ausgewählten HTAs von Ho et al. (2007) wurden in einem nächsten Schritt aktuellere Publikationen zur Kosteneffektivität in den Datenbanken MEDLINE und EMBASE gesucht.

Die in die Evaluation eingeschlossenen Primärpublikationen (aus HTAs und systematischen Reviews sowie aus identifizierten Primärpublikationen) wurden anhand des 56 Punkte umfassenden Kriterienkatalogs zur methodischen Qualität gesundheitsökonomischer Studien bewertet (s. Tab. 12). Dieser Kriterienkatalog wurde von den gesund heitsökonomischen Projektgruppen München, Hannover und Ulm im Konsensusverfahren erstellt (Checkliste 3) (Siebert et al. 1999a; Siebert et al. 1999b).

Es erfolgte eine Datenextraktion der in den eingeschlossenen Primärpublikationen angegeben Kosteneffektivitätsdaten unter besonderer Berücksichtigung von Subgruppenanalysen.

Währungskonversionen in Euro erfolgten über Bruttoinlandsprodukt-Kaufkraftparitäten (BIP KKP) des jeweiligen Jahres (OECD Health Data 2009). Euroangaben aus dem Ausland wurden ebenfalls mittels KKP in „deutsche“ Euro umgerechnet. Eine Inflationsbereinigung mittels des allgemeinen Verbraucherpreisindexes (VPI) wurde auf das Jahr 2007 vorgenommen. ${ }^{8}$

\subsubsection{Kostensensible Leitlinie}

Die Empfehlungen der ausgewählten klinischen Leitlinie für bestimmte Subgruppen von Patienten zur Implantation eines ICDs im Rahmen der Primärprävention zur Verhinderung des plötzlichen Herztodes bei Patienten mit chronischer Herzinsuffizienz wurden um Daten zur Kosteneffektivität dieser Subgruppen ergänzt.

8 https://www.destatis.de/DE/ZahlenFakten/GesamtwirtschaftUmwelt/Preise/Verbraucherpreisindizes/Tabellen_/VerbraucherpreiseKategorien.htm (Zugriff am 21.04.2015) 
4 Entwicklung einer Kostensensiblen Leitlinie zur Implantation eines ICDs bei Patienten mit chronischer Herzinsuffizienz

\subsubsection{Ergebnisse der Literaturrecherche}

\section{Klinische Leitlinien}

Das Ergebnis der Leitlinienrecherche kann Abbildung 3 entnommen werden.

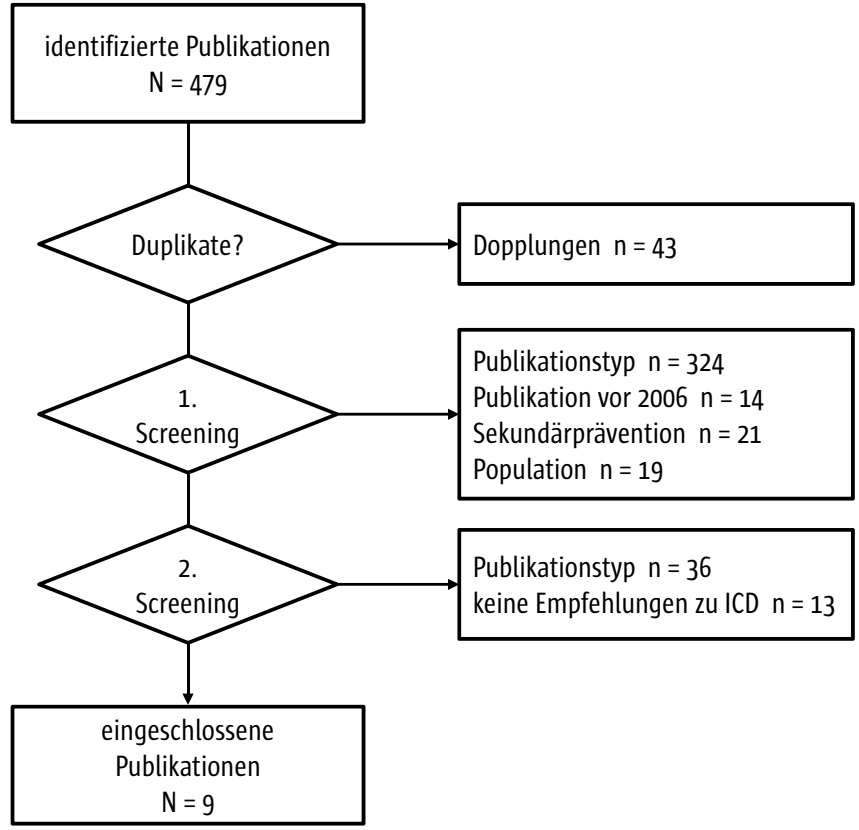

Abb. 3 Flussdiagramm zur Leitlinienrecherche

Im Rahmen der Literaturrecherche nach Leitlinien konnten 479 Publikationen identifiziert werden. Nach Ausschluss von 43 Dubletten konnten im Rahmen des 1. Screening 436 Publikationen aufgrund der festgelegten Ein- und Ausschlusskriterien durch Sichtung des Titels und Abstracts ausgeschlossen werden. Die verbliebenen 58 Referenzen wurden hinsichtlich der Ein- und Ausschlusskriterien im Volltext auf inhaltliche Relevanz geprüft (2. Screening). Im Ergebnis wurden 49 Dokumente im Rahmen des 2. Screenings ausgeschlossen. Im Ergebnis konnten 9 Publikationen eingeschlossen werden.

Für die Bewertung der 9 klinischen Leitlinien erfolgte von zwei voneinander unabhängigen Reviewern eine Untersuchung mithilfe des DELBI (Beyer et al. 2008), welches die Bewertung der methodischen Qualität medizinischer Leitlinien ermöglichen soll. Die g eingeschlossenen Leitlinien werden in Tabelle 10 (geordnet nach Publikationsjahr) aufgeführt.

Die Leitlinie von Dickstein et al. (2008) wurde als aktuellste und zudem mit dem höchsten Summenwert nach DELBI bewertete Leitlinie als Grundlage der Entwicklung einer KSLL ausgewählt. 
Tab. 10 Eingeschlossene Leitlinien

\begin{tabular}{|c|c|}
\hline Publikationsjahr & Leitlinie \\
\hline 2008 & $\begin{array}{l}\text { Epstein et al. } 2008 \\
\text { Dickstein et al. } 2008\end{array}$ \\
\hline 2007 & $\begin{array}{l}\text { SIGN } 2007 \\
\text { Institute for Clinical Systems Improvement (ICSI) et al. } 2007\end{array}$ \\
\hline 2006 & $\begin{array}{l}\text { National Heart Foundation of Australia and the Cardiac Society of Australia and } \\
\text { New Zealand } 2006 \\
\text { Zipes et al. } 2006 \\
\text { Adams et al. } 2006 \\
\text { Jung et al. } 2006 \\
\text { National Institute for Health and Clinical Excellence (NICE) } 2006\end{array}$ \\
\hline
\end{tabular}

\section{Medizinische Effektivität und Kosteneffektivität}

Das Ergebnis der HTA-Recherche kann Abbildung 4 entnommen werden.

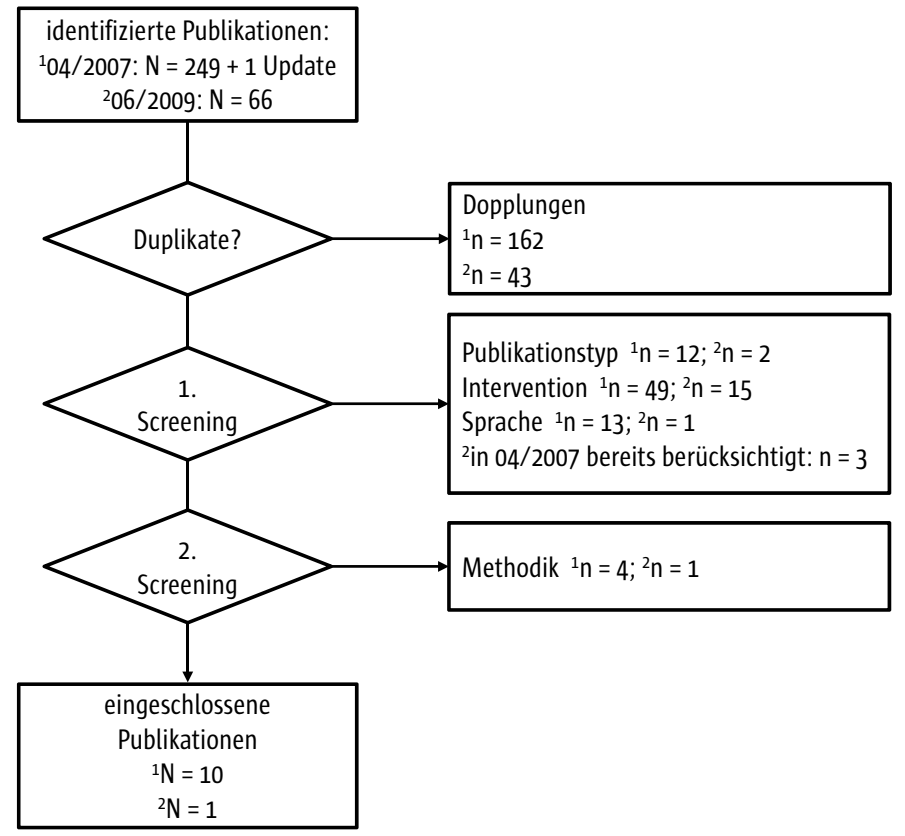

Abb. 4 Flussdiagramm zur HTA-Recherche 04/2007 und 06/2009

Es konnten im Rahmen der HTA-Recherche $04 / 2007 \mathrm{~N}=249+1$ Update und 06/2009 N = 66 Publikationen identifiziert werden. Die definierten Einschlusskriterien wurden benutzt, um anhand von Titel und Abstract die identifizier- 
4 Entwicklung einer Kostensensiblen Leitlinie zur Implantation eines ICDs bei Patienten mit chronischer Herzinsuffizienz

ten Literaturstellen von zwei Mitarbeitern unabhängig voneinander zu selektieren. Nach Ausschluss der Dubletten (04/2007: 162; 06/2009: 43) konnten im 1. Screening der HTA-Recherche $04 / 2007 \mathrm{n}=74$ und in der HTA-Recherche 06/2009 $n=21$ Publikationen ausgeschlossen werden. Im 2. Screening wurden nach Volltextsichtung in der HTA-Recherche $04 / 2007 \mathrm{n}=4$ und in der HTA-Recherche $06 / 2009 \mathrm{n}=1$ Publikationen aufgrund methodischer Mängel ausgeschlossen. Die in der HTA-Recherche eingeschlossenen to HTAs der Recherche 04/2007 und der eingeschlossene HTA der Recherche 06/2009 können Tabelle 11 entnommen werden.

Die in die Evaluation eingeschlossenen 11 Publikationen wurden anhand der Checklisten 1a [HTAs], 1b [systematische Reviews, Metaanalysen] geprüft. Die aktuellsten und qualitativ hochwertigsten HTAs von Ho et al. (2007) und von Van Brabandt et al. (2007) wurden im Ergebnis als Grundlage der weiteren Analysen zur medizinischen Effektivität und Kosteneffektivität verwendet.

Tabelle 12 können die in die Analyse der einbezogenen HTAs von Ho et al. und von Van Brabandt et al. eingeschlossenen Publikationen zur Kosteneffektivität entnommen werden.

Das Ergebnis der Literaturrecherche nach aktuelleren Publikationen zur Kosteneffektivität kann Abbildung 5 entnommen werden.

Nach Ausschluss von 151 Dubletten wurden im 1. Screening anhand von Titel und Abstract 61 Publikationen ausgeschlossen. Im 2. Screening konnten im Zuge der Volltextsichtung 332 Publikationen ausgeschlossen werden. Es wurden 6 Publikationen zur Kosteneffektivität in die weiteren Analysen eingeschlossen.

\section{Tab. 11 Eingeschlossene HTAs in Recherchen 04/2007 und 06/2009}

\section{Eingeschlossene Referenzen}

Recherche 04/2007

1. Ho et al. 2007. Einschluss der Literatur bis 25.01.2006.

2. Hancock et al. 2006

3. Buxton et al. 2006

4. Bryant et al. 2005

5. Bryant et al. 2004

6. The Medical Advisory Secretariat 2003

7. Brophy u. Costa 2004

8. Parkes et al. 2000

9. Hider 1997

10. The Medical Advisory Secretariat 2005 (Update of 2003 Review)

a ausgewählte HTAs als Grundlage für weitere Analysen zur medizinischen Effektivität und Kosteneffektivität

Recherche 06/2009

1. Van Brabandt et al. 2007. Einschluss der Literatur zur medizinischen Effektivität: 1. Juli 2003 - 8. Januar 2007; Einschluss der Literatur zur Kosteneffektivität bis Oktober 2006. ${ }^{\text {a }}$ 
Tab. 12 In HTAs eingeschlossene Publikationen zur Kosteneffektivität

\begin{tabular}{cc} 
Ho et al. 2007 & Van Brabandt et al. 2007 \\
Al-Khatib et al. 2005 & Al-Khatib et al. 2005 \\
\hline Anderson u. Camm 1993 & Chan et al. 2006 \\
\hline Chen u. Hay 2004 & Chen u. Hay 2004 \\
\hline Mushlin et al. 1998 & Goldenberg et al. 2005 \\
\hline Sanders et al. 2001 & Hancock et al. 2006 \\
\hline Sanders et al. 2004 & Mark et al. 2006 \\
\hline Sanders et al. 2005 & McGregor u. Chen 2004 \\
\hline & Mushlin et al. 1998 \\
\hline & Sanders et al. 2001 \\
\hline & Sanders et al. 2004
\end{tabular}

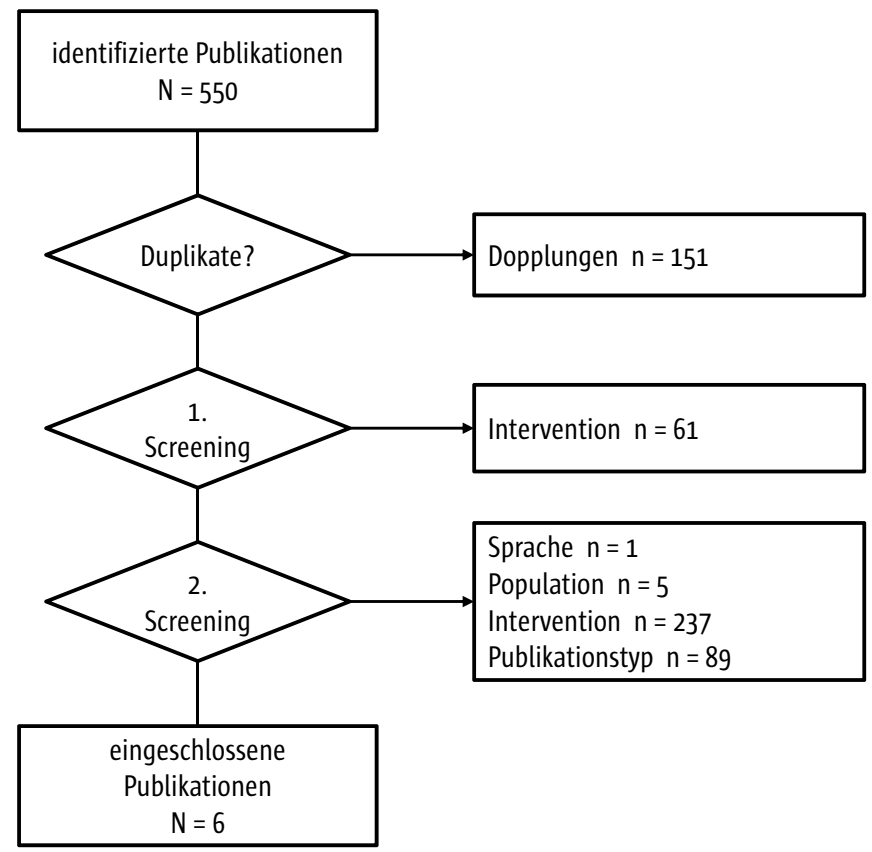

Abb. 5 Flussdiagramm zur Literaturrecherche nach aktuelleren Publikationen zur Kosteneffektivität 
4 Entwicklung einer Kostensensiblen Leitlinie zur Implantation eines ICDs bei Patienten mit chronischer Herzinsuffizienz

Tabelle 13 beinhaltet die in der Literaturrecherche nach aktuelleren Publikationen zur Kosteneffektivität eingeschlossenen 6 Referenzen.

\section{Tab. 13 Aktuellere Publikationen zur Kosteneffektivität}

Boriani et al. 2006

Bryant et al. 2007

Mark et al. 2006

Wang et al. 2008

You et al. 2007

Zwanziger et al. 2006

In den 6 gefundenen Referenzen wurde eine systematische Übersichtsarbeit von Bryant et al. (2007) identifiziert. In diesem Review wurde sowohl die Primärprävention als auch die Sekundärprävention des plötzlichen Herztodes durch Implantation eines ICDs untersucht. Tabelle 14 können die im systematischen Review von Bryant et al. enthaltenen Publikationen zur Kosteneffektivität in der Primärprävention des plötzlichen Herztodes mittels Implantation eines ICDs entnommen werden.

\section{Tab. 14 Im systematischen Review von Bryant et al. eingeschlossene Publikationen zur Kosteneffektivität}

\section{Bryant et al. 2007}

Mushlin et al. 1998

Sanders et al. 2001

Sanders et al. 2004

In der Zusammenfassung konnten durch die Literaturrecherche zur medizinischen Effektivität und Kosteneffektivität die in der folgenden Tabelle 15 auf-

\section{Tab. 15 Eingeschlossene Referenzen}

\begin{tabular}{ll} 
Referenz & Publikationstyp \\
Ho et al. 2007 & HTA \\
\hline Van Brabandt et al. 2007 & HTA \\
\hline Bryant et al. 2007 & Syst. Review \\
\hline Boriani et al. 2006 & Primärpublikation \\
\hline Mark et al. 2006 & Primärpublikation \\
\hline Wang et al. 2008 & Primärpublikation \\
\hline You et al. 2007 & Primärpublikation \\
\hline Zwanziger et al. 2006 & Primärpublikation
\end{tabular}


geführten Publikationen (mit dem jeweiligen Publikationstyp) eingeschlossen werden.

Im Rahmen der systematischen Literaturrecherche zur Kosteneffektivität des Einsatzes von implantierbaren Cardioverter/Defibrillatoren in der Primärprävention des plötzlichen Herztodes bei Patienten mit Herzinsuffizienz konnten (neben Alter, NYHA-Klassen, QRS-Komplex) bezüglich der Kosteneffektivität Subgruppenanalysen v.a. im Hinblick auf die Pumpfunktion der linken Herzkammer - gemessen über die linksventrikuläre Ejektionsfraktion (LVEF) - ermittelt werden.

\subsubsection{Ergebnisse der eingeschlossenen Publikationen}

\section{Klinische Leitlinien}

Dickstein et al. (2008) geben Empfehlungen zur Implantation eines ICDs in Abhängigkeit der LVEF für verschiedene Patientensubgruppen. Gemäß der Leitlinie von Dickstein et al. wird die Implantation eines ICDs im Rahmen der Primärprävention bei einer LVEF $\leq 35 \%$ empfohlen. Diese Empfehlung der Leitlinie basiert auf den Studienergebnissen der Sudden Cardiac Death in Heart Failure Trial (SCD-HeFT)-Studie. Die Empfehlungen der Leitlinie von Dickstein et al. (2008) können Tabelle 16 entnommen werden.

\section{Tab. 16 Empfehlungen der medizinischen Leitlinie von Dickstein et al. (2008)}

\begin{tabular}{lcc}
\hline LVEF & $\leq 35 \%$ & $>35 \%$ \\
\hline Empfehlung klinische Leitlinie (Dickstein et al. 2008) & $\checkmark$ & $\mathbf{x}$ \\
\hline
\end{tabular}

\section{Medizinische Effektivität und Kosteneffektivität}

Es konnten aus den oben genannten Publikationen 3 Referenzen identifiziert werden, die Subgruppenanalysen zur LVEF durchgeführt haben. Tabelle 17 zeigt die inkrementellen Kosten pro QALY der Publikationen von Mark et al.

Tab. 17 Inkrementelle Kosten/QALY Vergleich ICD vs. Kontrolle nach EF in Euro (Quelle der Daten: Mark et al. [2006], Sanders et al. [2001], Zwanziger et al. [2006]) (Währungskonversion mit OECD-Kaufkraftparitäten 2007)

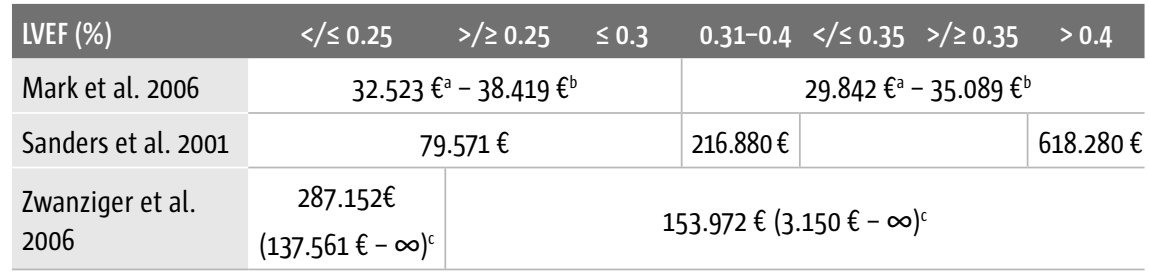

${ }^{a}$ nicht diskontiert; ${ }^{\text {b }}$ diskontiert mit 3\%; ' Angaben über 3,5 lahre 
4 Entwicklung einer Kostensensiblen Leitlinie zur Implantation eines ICDs bei Patienten mit chronischer Herzinsuffizienz

(2006), Sanders et al. (2001) sowie Zwanziger et al. (2006) für die Subgruppe Ejektionsfraktion (EF) auf.

Die beiden Publikationen von Mark et al. (2006) und von Zwanziger et al. (2006) wurden im Rahmen der exemplarischen KSLL nicht berücksichtigt, da anhand der Kosteneffektivitätsdaten keine Empfehlungen für eine KSLL generiert werden können; während bei Sanders et al. (2001) eine eindeutige Aussage für eine $\mathrm{EF}>0.30$ getroffen werden kann.

Der Modellierung von Sanders et al. 2001 zufolge hängt die Kosteneffektivität eines ICDs wesentlich von der Einschränkung der Pumpfunktion des Herzens ab. Bei einer $\mathrm{LVEF} \leq 30 \%$ betragen die zusätzlichen Kosten für ein hinzugewonnenes qualitätskorrigiertes Lebensjahr (QALY) beim Einsatz eines ICDs gegenüber medikamentöser Therapie (Amiodaron) etwa 80.00o Euro, bei einer LVEF zwischen 31\% und 40\% etwa 217.0oo € und bei einer LVEF > 40\% etwa 618.00o €. Bei einer stärker eingeschränkten Pumpfunktion des Herzens ist der therapeutische Vorteil eines ICDs gegenüber Amiodaron größer und damit das Verhältnis von Kosten zu Effekten günstiger. Tabelle 18 bietet eine Übersicht, wie sich in Abhängigkeit von der Pumpfunktion des Herzens das Sterberisiko der Patienten (als Maß für die Lebensbedrohlichkeit des Krankheitszustands), die Wirksamkeit von ICD und Amiodaron und die Kosteneffektivität eines ICDs im Vergleich zu Amiodaron unterscheiden.

\subsection{Kostensensible Leitlinie}

Um die Kostensensible Leitlinie erstellen zu können, wurden die Ergebnisse der Literaturrecherche zur medizinischen Effektivität/Kosteneffektivität mit der ermittelten Leitlinie zusammengeführt.

Auf der Grundlage der Daten zur Kosteneffektivität eines ICDs gegenüber der medikamentösen Behandlung mit Amiodaron (vgl. Tab. 18) kann nun die In-

Tab. 18 Darstellung der medizinischen Effektivität und Kosteneffektivität für LVEF (Quelle der Daten: Modellierung von Sanders et al. 2001) (Währungskonversion mit OECDKaufkraftparitäten 2007 [OECD Health Data 2009] und auf Tausender gerundet)

\begin{tabular}{llccc} 
& \multicolumn{3}{c}{ Linksventrikuläre Ejektionsfraktion (LVEF) } \\
\hline & $\leq 30 \%$ & $31 \%-40 \%$ & $>40 \%$ \\
\hline Sterblichkeitsraten nach 3 lahren & $34,7 \%$ & $20,6 \%$ & $8,6 \%$ \\
\hline Effektivität in QALYS & ICD & 6,23 & 7,87 & 10,29 \\
\hline & Amiodaron & 5,71 & 7,69 & 10,23 \\
\hline \multicolumn{1}{c}{ Differenz } & 0,52 & 0,18 & 0,06 \\
\hline $\begin{array}{l}\text { Zusatzkosten in } € / \text { QALY bei Einsatz eines } \\
\text { ICDs statt Amiodaron }\end{array}$ & 80.000 & 217.000 & 618.000 \\
\hline
\end{tabular}


Tab. 19 Darstellung der Empfehlungen der klinischen Leitlinie und der KSLL

\begin{tabular}{|lcccc}
\hline LVEF & $\leq 30 \%$ & $31 \%-35 \%$ & $36 \%-40 \%$ & $>40 \%$ \\
\hline Empfehlung klinische Leitlinie (Dickstein et al. 2008) & $\checkmark$ & $\checkmark$ & $\times$ & $\times$ \\
\hline Empfehlung KSLL (basierend auf Sanders et al. 2001) & $\checkmark$ & $\times$ & $\times$ & $\times$ \\
\hline
\end{tabular}

dikation wie folgt eingeschränkt werden: Während bei einer LVEF unter 30\% gegenüber der medikamentösen Therapie Zusatzkosten von ca. 79.500 entstehen, liegen diese bei einer LVEF von über 30\% bei über $200.000 €$ pro gewonnenem QALY. In Deutschland existiert bislang kein Schwellenwert, jedoch sind Werte für über 50.0oo €/QALY in Anlehnung an Entscheidungen des NICE (2006) als kritisch anzusehen. Orientiert man sich an diesen 50.000 €/QALY erscheinen bereits die Zusatzkosten bei einer LVEF von unter 30\% hoch, die Zusatzkosten bei einer LVEF von über 30\% übersteigen diesen Wert jedoch um ein Vielfaches. Dementsprechend würde eine Implantation eines ICDs nach der hier vorliegenden Kostensensiblen Leitlinie lediglich bei einer LVEF $\leq 30 \%$ vorgenommen, d.h. bei denjenigen Patienten, die eine stärker eingeschränkte Pumpfunktion des Herzens und einen größeren Nutzengewinn (o,52 QALYs) von einem ICD gegenüber der Behandlung mit Amiodaron haben. Patienten mit einer LVEF > 30\% sind weniger schwer erkrankt, haben ein niedrigeres Sterblichkeitsrisiko und einen deutlich geringeren Nutzengewinn von einem ICD $(\leq 0,18$ QALYs) und können ggf. medikamentös mit Amiodaron behandelt werden.

Tabelle 19 zeigt die Kostensensible Einschränkung der Indikation im Vergleich zur Empfehlung in der klinischen Leitlinie.

\subsection{Diskussion und Schlussfolgerung}

Im Rahmen der zunehmenden Diskussion zu den Themen Rationalisierung, Priorisierung und Rationierung im Gesundheitswesen finden sich Vorschläge, zur Entlastung des Arzt-Patienten-Verhältnisses eine Rationierung auf expliziter Ebene stattfinden zu lassen. Eine methodische Möglichkeit hierzu besteht in der Erarbeitung von Kostensensiblen Leitlinien (KSLL). Im Rahmen dieser Arbeit wurde beispielhaft eine KSLL zur Implantation eines ICDs in der Primärprävention des plötzlichen Herztodes bei Patienten mit chronischer Herzinsuffizienz entwickelt.

An diesem Beispiel konnte aufgezeigt werden, wie sich mögliche Unterschiede in der Empfehlung von klinischen Leitlinien und KSLL darstellen und somit im Sinne eines potenziellen Instrumentes der expliziten Rationierung wirken könnten.

Die hier vorgestellte KSLL präsentiert aufgrund inhaltlicher und methodischer Limitationen lediglich exemplarisch ein mögliches Szenario. 
4 Entwicklung einer Kostensensiblen Leitlinie zur Implantation eines ICDs bei Patienten mit chronischer Herzinsuffizienz

Die vorliegende KSLL basiert auf einer nicht vollständigen Literaturdarstellung. Die primäre Betrachtung der aktuellsten und qualitativ hochwertigsten HTAs und die im Jahre 2008 durchgeführte Literaturrecherche nach aktuelleren Publikationen zur Kosteneffektivität bilden nicht die insgesamt verfügbare Literatur zur Thematik der Implantation eines ICDs in der Primärprävention zur Verhinderung des plötzlichen Herztodes bei Patienten mit chronischer Herzinsuffizienz ab. Darüber hinaus wurden die Publikationen von Mark et al. (2006) und von Zwanziger et al. (2006) zur Kosteneffektivität im Rahmen der exemplarischen KSLL nicht berücksichtigt, da anhand der Kosteneffektivitätsdaten keine Empfehlungen für eine KSLL generiert werden können, während bei Sanders et al. eine eindeutige Aussage für eine $\mathrm{EF}>0.30$ getroffen werden konnte.

Grundsätzlich hat sich gezeigt, dass die Erstellung einer KSLL methodisch durchführbar ist. Die Erarbeitung Kostensensibler Leitlinien als Instrument der expliziten Leistungsbegrenzung erfordert jedoch einen hohen Zeit- und Ressourcenaufwand. Abhängig vom medizinischen Themenbereich und der hierzu verfügbaren publizierten Evidenz sind aufwendige Literaturrecherchen, Literaturselektionen, Datenextraktionen sowie Datensynthesen notwendig.

Im Hinblick auf die Qualität der zugrundeliegenden medizinischen Leitlinien, der Existenz/Verfügbarkeit ausreichender Daten, der Vergleichbarkeit vorhandener Daten zur Kosteneffektivität und der Aktualität der KSLL können jedoch die folgenden Probleme auftauchen. Interventionsspezifische Leitlinien sind nicht immer qualitativ hochwertig verfasst. Voraussetzung für die Erstellung von KSLL sind als Crundlage jedoch qualitativ hochwertige interventionsspezifische Leitlinien, die um gesundheitsökonomische Daten erweitert werden. Wünschenswert für die Erstellung einer KSLL für das deutsche Gesundheitssystem wäre eine vorhandene Leitlinie mit allen Elementen einer systematischen Entwicklung im Sinne einer S3-Leitlinie der Arbeitsgemeinschaft der Wissenschaftlichen Medizinischen Fachgesellschaften (AWMF) als medizinische Grundlage.

Die Erstellung von KSLL erfordert die Existenz von Daten zur Kosteneffektivität bei spezifischen Subgruppen von Patienten analog der klinischen Subgruppen in der medizinischen Leitlinie. Die Existenz dieser Daten zu Subgruppen konnte im vorliegenden Beispiel der Implantation eines ICDs im Rahmen der Primärprävention aufgezeigt und analysiert werden, kann aber nicht generell in allen medizinischen Bereichen gewährleistet werden.

Es ist anzumerken, dass weiterhin ein großer Forschungsbedarf im Bereich der ökonomischen Evaluation in Deutschland besteht. Um Empfehlungen unter Kosteneffektivitätsgesichtspunkten für das deutsche Gesundheitswesen generieren zu können, wäre das Vorhandensein ökonomischer Evaluationen aus Deutschland unabdingbar. Diese sind bislang jedoch noch nicht in ausreichender Anzahl verfügbar. 
In vielen Fällen stammen die Daten zur Kosteneffektivität der relevanten Subgruppen aus unterschiedlichen Ländern, sodass die Übertragbarkeit international erhobener Kosteneffektivitätsdaten auf den deutschen Versorgungskontext zu prüfen und ggf. eine eigenständige Adaptation und Berechnung notwendig ist.

Ein Gütekriterium der KSLL stellt die Aktualität dar. Aufgrund der sich dynamisch entwickelnden Gesundheitskosten muss die Aktualität von KSLL regelmäßig überprüft und zwingend in kontinuierlichen Abständen angepasst werden.

Im Rahmen der vorliegenden Arbeit konnte exemplarisch die Möglichkeit der Erstellung einer Kostensensiblen Leitlinie gezeigt werden. Aufgrund der oben aufgezeigten Problemfelder sowie des notwendigen Ressourceneinsatzes zur Erstellung einer KSLL ist noch nicht abzusehen, inwiefern dieses Instrument Eingang in die Versorgungspraxis finden kann.

\section{Literatur}

Adams KG, Lindenfeld I, Arnold JMO, Baker DW, Barnard DH, Baughman KL, et al. HFSA 2006 Comprehensive Heart Failure Practice Guideline. I Cardiac Failure 2006; 12: e1-e122

Al-Khatib SM, Anstrom KJ, Eisenstein EL, Peterson ED, Jollis |G, Mark DB, et al. Clinical and Economic Implications of the Multicenter Automatic Defibrillator Implantation Trial-II. Ann Intern Med 2005; 142: 593-600

Anderson MH, Camm A). Implications for present and future applications of the implantable cardioverter-defibrillator resulting from the use of a simple model of cost efficacy. Br Heart | 1993; 69: 83-92

Beyer $M$ et al. Deutsches Instrument zur methodischen Leitlinien-Bewertung (DELBI) Fassung 2005/2006 + Domäne 8. Arbeitsgemeinschaft der Wissenschaftlichen Medizinischen Fachgesellschaften e.V.; 2008. URL: http://www.awmf.org/fileadmin/user_upload/Leitlinien/Werkzeuge/delbi-fassung-2005-2006-domaene-8-2008-1.pdf (Zugriff am 21.04.2015)

Boriani G, Biffi M, Russo M, Lunati M, Botto G, Proclemer A, et al. Primary Prevention of Sudden Cardiac Death: Can We Afford the Cost of Cardioverter-Defibrillators? Data from the Search-MI Registry-Italian Sub-study. Pace 2006; 29: S29-S34

Brophy I, Costa V. The Use of Biventricular Pacemakers at the McGill University Health Centre. 2004. URL: http:// www.mcgill.ca/files/tau/biventricular_pacemaker_final_report1.pdf (Zugriff am 21.04.2015)

Bryant I, Brodin H, Loveman E, Clegg A. Clinical effectiveness and cost-effectiveness of implantable cardioverter defibrillators for arrhythmias: A systematic review and economic evaluation. Int I Technol Assess Health Care 2007; 23 (1): 63-70

Bryant I, Brodin H, Loveman E, Payne E, Clegg A. The clinical and cost-effectiveness of implantable cardioverter defibrillators: a systematic review. Health Technol Asses 2005; 9 (36): 1-150

Bryant I, Brodin H, Loveman E, Payne E, Clegg A. The clinical effectiveness and cost effectiveness of implantable cardioverter defibrillators. arrhythmias 2004 (Vorläufer des HTA von 2005, s. Bryant et al. 2005)

Buxton M, Caine N, Chase D, Connelly D, Grace A, Jackson C, et al. A review of the evidence on the effects and costs of implantable cardioverter defibrillator therapy in different patient groups, and modelling of costeffectiveness and cost-utility for these groups in a UK context. Health Technol Assess 2006; 10 (27): 1-164

Chan PS, Stein K, Chow T, Fendrick M, Bigger IT, Vijan S. Cost-Effectiveness of a Microvolt T-Wave Alternans Screening Strategy for Implantable Cardioverter-Defibrillator Placement in the MADIT-II-Eligible Population. I Am Coll Cardiol 2006; 48: 112-21

Chen L, Hay JW. Cost-Effectiveness of Primary Implanted Cardioverter Defibrillator for Sudden Death Prevention in Congestive Heart Failure. Cardiovascular Drugs and Therapy 2004; 18: 161-70 
Dickstein K, Cohen-Solal A, Filippatos G, et al. ESC Guidelines for the diagnosis and treatment of acute and chronic heart failure 2008: the Task Force for the Diagnosis and Treatment of Acute and Chronic Heart Failure 2008 of the European Society of Cardiology. Developed in collaboration with the Heart Failure Association of the ESC (HFA) and endorsed by the European Society of Intensive Care Medicine (ESICM). Eur Heart J 2008; 29 (19): 2388-442

Eccles M, Mason J. How to develop cost-conscious guidelines. Health Technol Assess 2001; 5 (16): 1- 69

Epstein AE, Dimarco JP, Ellenbogen KA, Estes NA, III, Freedman RA, Gettes LS, et al. ACC/AHA/HRS 2008 Guidelines for device-based therapy of cardiac rhythm abnormalities. Heart Rhythm 2008; 5 (6): e1-62

Goldenberg I, Moss A), Maron BJ, Dick AW, Zareba W. Cost-Effectiveness of Implanted Defibrillators in Young People with Inherited Cardiac Arrhythmias. Ann Noninvasive Electrocardiol 2005; 10 (4): 67-83

HancockS, HiderP, Weir R, BidwellS, HoganS, AliW, etal. MSACreference 32, Implantablecardioverter defibrillators for prevention of sudden cardiac death. Medical Services Advisory Committee, Australia 2006. URL: http:// www.msac.gov.au/internet/msac/publishing.nsf/Content/8FD1D98FE64C8A2FCA2575AD0082FD8F/\$File/ MSAC\%20Ref\%2032\%20-\%20ICDs.pdf (Zugriff am 21.04.2015)

Hider P. Outcomes from the use of the Implantable Cardiac Defibrillator - A critical appraisal of the literature. 1997. URL: http://nzhta.chmeds.ac.nz/publications/nzhta1.pdf (Zugriff am 21.04.2015)

Ho C, Li H, Noorani H, Cimon K, Campbell K, Tang A, Birnie D. Implantable cardiac defibrillators for primary prevention of sudden cardiac death in high risk patients: a meta-analysis of clinical efficacy, and a review of cost-effectiveness and psychosocial issues [Technology report no 81]. Ottawa: Canadian Agency for Drugs and Technologies in Health 2007. URL: http://www.cadth.ca/media/pdf/332_ICD_tr_e.pdf (Zugriff am 21.04.2015)

Institute for Clinical Systems Improvement (ICSI), Kopecky S, Festin R, Smars P, Fareed MT, 0jha A, et al. Health Care Guideline: Heart Failure in Adults. 2007

Jung W, Andresen D, Block M, Bocker D, Hohnloser SH, Kuck KH, et al. [Guidelines for the implantation of defibrillators.] Leitlinien zur Implantation von Defibrillatoren. Clin Res Cardiol 2006 Dec; 95 (12): 696-708

Mark DB, Nelson CL, Anstrom KJ, Al-Khatib SM, et al. Cost-Effectiveness of Defibrillator Therapy or Amiodarone in Chronic Stable Heart Failure. Results From the Sudden Cardiac Death in Heart Failure Trial (SCD-HeFT). Circulation 2006; 114: 135-142

McGregor M, Chen I. Should the implantable cardiac defibrillator be used for primary prevention of sudden death? A review of the issues relevant to hospital decision making. Canadian Journal of Cardiology 2004; 20 (12): 1199-204

Mushlin Al, Hall WI, Zwanziger I, Gajary E, Andrews M, Marron R, et al. The Cost-effectiveness of Automatic Implantable Cardiac Defibrillators: Results From MADIT. Circulation 1998; 97: 2129-35

National Heart Foundation of Australia and the Cardiac Society of Australia and New Zealand. Guidelines for the prevention, detection and management of chronic heart failure in Australia. 2006

National Institute for Health and Clinical Excellence (NICE). Implantable defibrillators for arrhythmias. Review of Technical Appraisal 11; Technology Appraisal 95. 2006

OECD Health Data 2009. PPPs and exchange rates. URL: http://stats.oecd.org/Index.aspx?datasetcode=SNA_TABLE4 (Zugriff am 21.04.2015)

Parkes |, Bryant |, Milne R. Implantable cardioverter defibrillators: arrhythmias. A rapid and systematic review. Health Technol Assess 2000; 4 (26):1-69

Sanders GD, Hlatky MA, Every NR, McDonald KM et al. Potential Cost-Effectiveness of Prophylactic Use of the Implantable Cardioverter Defibrillator or Amiodarone after Myocardial Infarction. Ann Intern Med 2001; 135 (10): 870-883

Sanders GD, Hlatky MA, Owens DK, Aronson N, Ziegler KM, et al. Special Report: Cost-Effectiveness of Implantable Cardioverter-Defibrillators in a MADIT-II Population. Assessment Program 2004; 19 (3)

Sanders GD, Hlatky MA, Owens DK. Cost-Effectiveness of Implantable Cardioverter-Defibrillators. The New England Jorunal of Medicine 2005; 353 (14): 1471-80

Siebert U, Behrend C, Mühlberger N, Wasem I, Greiner W, Graf von der Schulenburg JM, Welte R, Leidl R. Entwicklung eines Kriterienkatalogs zur Beschreibung und Bewertung ökonomischer Evaluationsstudien in 
Deutschland. In: Leidl R, Graf von der Schulenburg JM, Wasem I (eds) Ansätze und Methoden der ökonomischen Evaluation Eine internationale Perspektive. Nomos Verlag, Baden-Baden, 1999a

Siebert U, Mühlberger N, Behrend C, Wasem I. Technology Assessment for Health Care: A tool for explicitly evaluating economic studies. In: Medizinische Informatik, Biometrie und Epidemiologie. MMV Medizin, München, 1999b: 142-143

SIGN. Scottish Intercollegiate Guidelines Network. 94 - Cardiac arrhythmias in coronary heart disease - A national clinical guideline. 2007. URL: http://www.sign.ac.uk/pdf/signg4.pdf (Zugriff am 21.04.2015)

The Medical Advisory Secretariat (Ontario). Implantable Cardioverter Defibrillator (ICD) - Prophylactic Use. 2003. URL: http://www.ontla.on.ca/library/repository/mon/8000/245557.pdf (Zugriff am 21.04.2015)

The Medical Advisory Secretariat (Ontario). Implantable Cardioverter Defibrillator (ICD) - Prophylactic Use. 2005 (Update of 2003 Review): Ontario Health Technology Assessment Series 2005回 5 (14)

Van Brabandt H, Thiry N, Neyt M, et al. The Implantable Cardioverter Defibrillator: A Health Technology Assessment. Health Technology Assessment (HTA). Brüssel: Belgian Health Care Knowledge Centre (KCE). 2007. URL: https://kce.fgov.be/sites/default/files/page_documents/d20071027323.pdf (Zugriff am 21.04.2015)

Wang K, Yamauchi K, Li P, Kato H, Kobayashi M, Kato K, et al. Cost-Effectiveness of Implantable CardioverterDefibrillators in Brugada Syndrome Treatment. I Med Syst 2008; 32: 51-57

You II, Woo A, Ko DT, Cameron DA, Mihailovic A, Krahn M. Life expectancy gains and cost-effectiveness of implantable cardioverter/defibrillators for the primary prevention of sudden cardiac death in patients with hypertrophic cardiomyopathy. Am Heart I 2007; 154: 899-907

ZEKO (o.V.) Stellungnahme der Zentralen Kommission zur Wahrung ethischer Grundsätze in der Medizin und ihren Grenzgebieten (Zentrale Ethikkommission) bei der Bundesärztekammer zur Priorisierung medizinischer Leistungen im System der Gesetzlichen Krankenversicherung (GKV). Langfassung 2007. URL: http:// www.zentrale-ethikkommission.de/downloads/LangfassungPriorisierung.pdf (Zugriff am 21.04.2015)

Zipes DP, Camm Al, Borggrefe M, Buxton AE, Chaitman B, Fromer M, et al. ACC/AHA/ESC 2006 guidelines for management of patients with ventricular arrhythmias and the prevention of sudden cardiac death: a report of the American College of Cardiology/American Heart Association Task Force and the European Society of Cardiology Committee for Practice Guidelines (Writing Committee to Develop Guidelines for Management of Patients With Ventricular Arrhythmias and the Prevention of Sudden Cardiac Death). I Am Coll Cardiol 2006 Sep 5; 48 (5): e247-e346

Zwanziger J, Hall W], Dick AW, Zhao Het al. The Cost Effectiveness of Implantable Cardioverter-Defibrillators Results from the Multicenter Automatic Defibrillator Implantation Trial (MADIT)-II. I Am Coll Cardiol 2006; 47 (11): 2310-2318 